Jean-Loïc LE QUELLEC, Bernard SERGENT (éds.), Dictionnaire critique de mythologie

Paris, CNRS Éditions, 2017, xx-1554 p.

Jean-Bruno Renard

\title{
CpenEdition
}

Journals

Édition électronique

URL : https://journals.openedition.org/assr/45020

DOI : $10.4000 /$ assr.45020

ISSN : $1777-5825$

Éditeur

Éditions de l'EHESS

Édition imprimée

Date de publication : 1 décembre 2018

Pagination : 324-326

ISSN : 0335-5985

Référence électronique

Jean-Bruno Renard, « Jean-Loïc LE quelLec, Bernard SERGENT (éds.), Dictionnaire critique de mythologie», Archives de sciences sociales des religions [En ligne], 184 | octobre-décembre 2018, mis en ligne le 01 décembre 2018, consulté le 10 janvier 2022. URL : http://journals.openedition.org/assr/45020 ; DOI : https://doi.org/10.4000/assr.45020

Ce document a été généré automatiquement le 10 janvier 2022.

(c) Archives de sciences sociales des religions 


\section{Jean-Loïc LE QUELLEC, Bernard SERGENT (éds.), Dictionnaire critique de mythologie}

Paris, CNRS Éditions, 2017, xX-1554 p.

Jean-Bruno Renard

\section{RÉFÉRENCE}

Jean-Loïc LE QUeLLEC, Bernard SERGENT (éds.), Dictionnaire critique de mythologie, Paris, CNRS Éditions, 2017, XX-1554 p.

1 Le terme «mythologie »- comme les mots «folklore » et « mathématiques » d'ailleurs - a l'inconvénient de désigner en même temps un objet de recherche et la science qui étudie cet objet. De très nombreux livres et articles présentent des «matériaux mythiques", qu'ils soient anciens (notamment les mythes des Antiquités égyptienne, mésopotamienne, grecque, romaine, celtique) ou toujours actifs comme la Bible, issus de cultures écrites ou de cultures orales, comme par exemple les mythes africains. Le travail de Jean-Loïc Le Quellec et Bernard Sergent se distingue de ces publications en proposant « un tableau d'ensemble de la science mythologique» (p. IX). Unique en son genre, ce livre volumineux est un ouvrage indispensable pour tous les chercheurs désireux d'acquérir, de compléter ou d'approfondir la connaissance des grands auteurs, des théories, des concepts et des méthodes de la science mythologique. Présentées par ordre alphabétique, les quelque 1400 entrées du dictionnaire relèvent de trois catégories : les concepts utilisés dans la science mythologique, les auteurs qui s'y sont distingués et les thèmes mythiques les plus fréquents.

2 Comme il se doit pour un dictionnaire de sciences humaines, la part du lion est accordée aux concepts, qui représentent environ 900 notices, soit $63 \%$ des entrées. L'attention portée au lexique utilisé pour l'étude des mythes est d'autant plus justifiée que les notions sont nombreuses, de natures variées, d'usage inégal et, souvent, de 
définitions différentes selon les auteurs. On y trouve bien sûr les termes désignant les divers genres narratifs proches du Mythe (dans cette recension, les noms d'entrées sont en italique, sauf pour les mythologues): Conte et Légende, mais aussi Épopée, Exemplum, Fable, Mémorat, Roman, Rumeur... D'autres notions traitent de la structure du récit mythique : Structure, Invariants, Variante, Version, Motif et Allomotif (quand deux motifs différents jouent le même rôle dans deux versions d'un même récit, par exemple un chat dans une version est remplacé par un scarabée dans une autre; cette notion essentielle permet d'établir une équivalence symbolique entre deux motifs et éclaire leur signification), Thèmes, Types, Séquences, Codes (les codes acoustique, alimentaire, astronomique, économique, de parenté, etc., qui, selon Claude Lévi-Strauss, constituent la «structure feuilletée» du mythe), Héros, Épreuves, Actant (rôle joué par un personnage), Personnage, Rôle, Oppositions binaires, Médiation... D'autres notions encore concernent les mécanismes de transformation du contenu du mythe: Actualisation, Amplification, Chiasme, Condensation, Dédoublement, Euphémisation, Inversion, Localisation, Permutation, Rationalisation, Réduction... Enfin, des entrées présentent les diverses théories traitant de l'origine, de la transmission, de la répartition et de la fonction des mythes, notamment: Allégorisme, Astrale (théorie), Diffusionnisme, Évhémérisme, Fonctionnalisme, Indianiste (théorie), Naturalisme, Psychanalyse, Ritualiste (théorie), Structuralisme. L'entrée Comparatisme rappelle qu'il n'y a de science mythologique que comparée, quelle que soit l'étendue du cadre de comparaison. L'entrée École ne recense pas moins de 35 écoles de pensée qui prétendent expliquer la formation des mythes et les interpréter. Cette belle synthèse historique des recherches sur les mythes fait le lien entre les concepts et les auteurs.

Le Dictionnaire présente 168 mythologues, qui font chacun l'objet d'une notice biobibliographique. Antti Aarne, Franz Boas, Henri Dontenville, Georges Dumézil, James Frazer, les frères Grimm, Luc de Heusch, Claude Lévi-Strauss, Friedrich-Max Müller, Vladimir Propp, Jean-Pierre Vernant: tous les grands noms sont là, mais aussi beaucoup d'auteurs peu connus ou méconnus. Des précurseurs de la science des mythes sont signalés : l'Islandais Snorri Sturluson qui, au Moyen Âge, recueillit la mythologie nordique ; l'érudit italien du xvI siècle Lilio Giraldi qui démêla la mythologie grecque et la mythologie égyptienne, jusque-là confondues; Fontenelle, dont l'ouvrage De l'origine des fables, au début du XviII ${ }^{\mathrm{e}}$ siècle, peut être considéré comme le premier essai sur la formation et la fonction des mythes.

On peut tirer quelques observations de l'examen des profils des mythologues. L'étude des mythes étant une spécialisation plus qu'une discipline institutionnalisée, il n'est pas étonnant que les mythologues appartiennent à diverses sciences humaines qui traitent de matériaux mythiques: historiens des religions, ethnologues, anthropologues, folkloristes, linguistes (philologues, sémiologues), psychanalystes (10 auteurs), antiquisants (les hellénistes sont très nombreux, mais aussi les celtisants) et orientalistes (indianistes, iranistes...). La nationalité des mythologues reflète des traditions intellectuelles fortes dans l'étude des mythes: on compte 47 Français (on ne peut écarter un biais dû aux auteurs du Dictionnaire, tous deux français, mais il est vrai aussi que la France du $\mathrm{xx}^{\mathrm{e}}$ siècle a donné à la science des mythes ses deux plus beaux fleurons: Georges Dumézil et Claude Lévi-Strauss); 35 Allemands, en raison de l'importance de l'anthropologie, de la philologie et du folklore outre-Rhin, notamment au XIX siècle; 32 auteurs anglo-saxons (Anglais, Américains, Écossais); mais il faut aussi souligner la présence de 17 mythologues issus des pays nordiques (Suède, 
Finlande et Danemark principalement), qui sont à l'origine d'avancées significatives dans l'étude des contes et des légendes. Malgré la difficulté de langue, les auteurs du Dictionnaire ont inclus deux mythologues russes et deux mythologues japonais.

5 Si la présence de Durkheim parmi les mythologues est justifiée, on peut regretter l'absence de deux durkheimiens qui ont beaucoup apporté à l'étude des mythes: Maurice Halbwachs, dont la notion de "mémoire collective » a été illustrée dans sa Topographie légendaire des Évangiles en Terre Sainte (1941), et Robert Hertz, dont plusieurs textes ont été réunis dans l'ouvrage posthume Sociologie religieuse et folklore (1928). Roger Bastide, qui a rédigé l'article « La mythologie » dans le volume Ethnologie générale (Gallimard, Pléiade, 1968), aurait également pu être l'objet d'une notice. Jean-Loïc Le Quellec et Bernard Sergent ont pris le risque de faire entrer huit auteurs de leur vivant dans le panthéon des mythologues. On ne contestera pas leur choix, car l'œuvre déjà bien établie de ces auteurs, dont le plus «jeune» est né en 1950, plaide pour leur reconnaissance. Mais, inévitablement, il pourra être reproché aux auteurs du Dictionnaire de ne faire figurer qu'en bibliographie des chercheurs qui auraient mérité une notice, par exemple la folkloriste Nicole Belmont ou le médiéviste Philippe Walter.

En complément des notices consacrées aux mythologues, le Dictionnaire propose une imposante bibliographie de 173 pages, soit quelque 6500 références correspondant à environ 3400 auteurs différents. Cette bibliographie d'ouvrages, de contributions dans des ouvrages collectifs et d'articles de revues constitue une source documentaire précieuse pour les études mythologiques.

7 Les notices thématiques représentent environ 340 entrées, soit $25 \%$ du total. Avec érudition, puisque les mythes de près de 1400 ethnies différentes sont évoqués, le Dictionnaire nous invite à une promenade dans le jardin des mythes. En allant des thèmes les plus généraux aux thèmes les plus précis, on peut distinguer trois types de thèmes : les grandes catégories indissociables du mythe, les thèmes mythiques « à large répartition » et les motifs narratifs.

8 Les thèmes les plus généraux donnent lieu à des articles développés où les différents aspects d'une question sont exposés, tout en renvoyant à des entrées en rapport avec le sujet principal. Citons quelques exemples. Les entrées Histoire et Temps évoquent notamment l'histoire des mythes, la question du lien entre l'historique et le mythique (Histoire mythifiée et mythologie historicisée), la spécificité du temps mythique (le temps primordial), la divinisation du Temps. Les entrées Géographie, Lieu et Paysage traitent des explications mythiques de l'espace ainsi que de la localisation des mythes, et surtout des légendes. À ce propos, on regrette l'absence d'une entrée "Pays mythiques et légendaires ». Les entrées Étymologie, Nom propre et Toponymie montrent à la fois l'intérêt et les risques de l'analyse étymologique des noms de dieux, de héros et de lieux, les explications savantes interférant parfois avec des fausses étymologies populaires. L'entrée Nombres traite à la fois des structures des contes et des mythes (par exemple les trois épreuves) et du riche symbolisme des nombres. L'entrée Musique évoque la place de la musique dans les mythes, les mythes d'origine de la musique et l'homologie, développée par Lévi-Strauss, entre un mythe et une partition musicale. L'entrée Politique souligne les fondements mythologiques des idéologies politiques. L'entrée Rêve rappelle la présence des rêves dans les mythes et l'équivalence établie par la psychanalyse entre le rêve et le mythe. L'entrée Roman fait le point sur les passages du mythe au roman (les épopées) et, inversement, du roman au mythe (Faust, Dracula). 
9 Les mythes «à large répartition » permettent une comparaison entre des récits mythiques différents associés à un même thème. La fonction première du mythe étant d'expliquer pourquoi les choses sont ce qu'elles sont, de nombreuses entrées du Dictionnaire sont consacrées aux mythes d'origine. Dans le domaine de la Nature: mythes d'origine du Monde (Cosmogonie), des Fleurs, des Fleuves, de la Foudre, de la Mer, de la Nuit, de la Pluie, des Séismes, du Tonnerre, du Vent... Dans le domaine de la biologie : mythes d'origine de l'Accouchement, de l'Anus, des Maladies, de la Mort, de la Sexualité (et de la différenciation sexuelle), du Pénis et du Vagin... Sur l'origine de l'homme et des peuples: Anthropogonie, Ethnogonie, Homme, Langues... Les mythes d'origine des techniques: Agriculture, Cuisine, Domestication, Écriture, Fer, Feu, Forgerons, Funéraires (coutumes), Masques, Musique (instruments), Poterie... Ces entrées passionnantes du Dictionnaire montrent à la fois des préoccupations universelles et la variété des réponses proposées par les mythes. Par exemple l'origine du Feu est déclinée selon deux modalités principales: le feu est volé à un être mythique ou bien l'homme doit reconquérir un feu perdu (éteint par le déluge ou par manque d'attention). La diversité des races et des peuples (Ethnogonie) est expliquée soit par la dispersion géographique d'hommes partis à la recherche d'objets perdus, soit par des frères issus d'un couple primordial mais qui vont être à l'origine des diverses branches de l'humanité. L'entrée Mort (19 pages, p. 820-838) est un petit bijou de mythologie comparée, listant une dizaine d'origines mythiques de la mortalité : la désobéissance à un ordre divin, une réponse faite à contretemps, un mauvais choix, un message perverti, un cri de peur, l'échec d'une tentative de résurrection, l'échec dans une épreuve, un agent extérieur, la détumescence du phallus, une querelle ou un meurtre original, une dette à payer, une punition.

10 Indissociable des mythes, le Héros est un personnage central qui présente, pour reprendre l'expression de Joseph Campbell, de multiples visages: Anti-héros, Héros culturel, Héros fondateur, Héros caché, Héros victime, Infirmités qualifiantes, Trickster...

11 Enfin, une série de mythes évoquent des catastrophes: Déluge, Désordre cosmique, Eschatologie, Fin du monde...

12 Les motifs narratifs, quant à eux, occupent une bonne place dans le Dictionnaire. Les mythologues les désignent le plus souvent par un intitulé associant un nom commun à une épithète inattendue. Ils suscitent ainsi le désir de connaître l'histoire et laissent entrevoir au lecteur toute la poésie fantastique des mythes, des contes et des légendes. Que l'on en juge par cette sélection de motifs : l'Aide miraculeuse, l'Auxiliaire animal ou le Guide animal, le Bourdon qui reverdit, le Changelin (enfant de fée substitué à un enfant humain), le Chasseur maudit, le Chaudron inépuisable (chaudron d'abondance ou chaudron de résurrection), la Conception miraculeuse et la Naissance miraculeuse, la Corde céleste ou l'Échelle céleste (permettant de monter au ciel), la Danseuse obscène, le Dénicheur d'oiseau, la Fille-cygne (femme se métamorphosant en oiseau), les Frères rivaux, la Gourde dévorante (en Afrique) ou créatrice (en Asie du Sud-Est), le Mort reconnaissant, l'Ogre stupide, le Passeur susceptible (un animal fait traverser un cours d'eau mais il ne faut pas le contrarier), le Rajeunissement trompeur (imitation fatale d'une magie de rajeunissement), la Précaution inutile, la Révolte des outils, les Statues récalcitrantes (qui refusent d'être déplacées), la Ville engloutie, le Voyage dans l'Autre-Monde (Au-delà, Ciel, Enfer). Ces motifs sont des épisodes narratifs qui se rencontrent avec une fréquence plus ou moins grande dans une ou plusieurs aires culturelles. Ils peuvent 
indifféremment être présents dans toutes sortes de récits : contes, mythes, légendes, épopées.

13 Ce Dictionnaire critique de mythologie montre l'unité et l'universalité de la pensée mythique ainsi que l'infinie variété des récits, l'examen des motifs et des thèmes permettant précisément de relier entre elles ces deux dimensions. Il ne fait aucun doute que le «Le Quellec-Sergent» est appelé à devenir un ouvrage de référence incontournable pour l'étude des mythes. 